\title{
INFLUÊNCIA DE FATORES HUMANOS NA QUALIDADE DA MALHA PARA ANTROPOMETRIA DIGITAL: TEMPO, POSTURA E CONFORTO
}

\section{INFLUENCE OF HUMAN FACTORS ON MESH QUALITY FOR DIGITAL ANTHROPOMETRY: TIME, POSTURE AND COMFORT}

\author{
Isabella de Souza Sierra ${ }^{1}$, M.Sc. \\ Matheus C. Barbosa ${ }^{2}$, Bach. \\ Bruno Rocha ${ }^{3}$, Bach. \\ Maria Lúcia Leite Ribeiro Okimoto ${ }^{4}$, D.Sc. \\ (1) Universidade Federal do Paraná \\ isabella.sierra@ufpr.br \\ (2) Universidade Federal do Paraná \\ cbarbosa.math@gmail.com \\ (3) Universidade Federal do Paraná \\ brunogr96@gmail.com \\ (4) Universidade Federal do Paraná \\ lucia.demec@ufpr.br
}

\begin{abstract}
Digitalização corporal 3D, Antropometria digital, Postura
Admitiu-se a hipótese de que ao usar escâneres de baixo custo, as principais influências na qualidade da malha são as posturas adotadas e o tempo de escaneamento e o conforto dos participantes que afetam a sua capacidade de manter a postura. Encontrou-se que as posturas adotadas tiveram mais influência na qualidade do que o tempo de digitalização.

$3 D$ body scanning, Digital anthropometry, Posture

We hypothesized that when using low-cost scanners, the main influences on the mesh quality are the postures adopted, the scanning time and the comfort of the participants that affect their ability to maintain the posture. We found that the postures adopted had more influence on quality than the digitization time.
\end{abstract}

\section{Introdução}

Ao realizar estudos de antropometria digital em larga escala, utilizando a digitalização $3 \mathrm{D}$, além da seleção de equipamento (KOUCHI, 2012) e software acompanhante, os pesquisadores devem considerar a participação do usuário (SCHWARZMUELLER; MARSHALL; SUMMERSKILL, 2018; SILVA; SALVALAIO; KINDLEIN , 2010) e também seu próprio trabalho de pós-processamento (HEYMSFIELD et al., 2018; CUI; STRICKER, 2011) pois estes afetam no tempo de efetivação e qualidade final do estudo.

Em termos da participação do usuário considera-se que está atrelada com a compreensão do protocolo de coleta, preparação do participante para o momento da digitailzação como orientação para o uso de roupas adequadas e de averiguação de sua capacidade de realizar as atividades planejadas como atingir a posição determinada e mantê-la pelo período necessário para a digitalização.

Já o trabalho de pós-processamento envolve a atividade do pesquisador de limpar a malha obtida com o escaneamento para a concretização das medidas e a efetiva realização dos cortes e marcações para a retirada das medidas. Nesse caso quanto menor a qualidade da malha coletada, ou seja, com mais falhas, buracos e distorções, maior é o trabalho do pesquisador e tempo dispendido.

Ainda, se considera que, no processo de digitalização, os participantes influenciam diretamente a qualidade da malha obtida e que a qualidade da malha é responsável pela maior parte do tempo de pós-processamento e pela qualidade das medições realizadas. Isso está ainda mais presente quando se utiliza hardware de baixo custo como o Kinect, devido ao seu tempo relativamente 
alto de escaneamento (BRAGANÇA et al., 2017) e suas limitações de qualidade (GUIDI; GONIZZI; MICOLI, 2016).

Embora o escâner Kinect seja uma opção útil para a realização de antropometria digital, ainda existem inúmeras limitações para o equipamento.

Especialmente, as restrições de tempo são relevantes porque, ao usar apenas um Kinect, os pesquisadores atingem um tempo de escaneamento entre 30 segundos e três minutos (BRENDLER et al., 2016; COSTA et al., 2015; GARSTHAGEN, 2014). O que implica na necessidade de o participante manter-se estático na posição determinada por este período.

Algumas dessas limitações podem ser atenuadas ao usar mais de um Kinect por vez, de modo a cobrir mais área da superfície corporal e, por fim, levar menos tempo a escaneamento (PARK; REED, 2014). No entanto, ao adicionar mais equipamentos Kinect ao estudo, surgem outras preocupações como o alinhamento das malhas (HIRSHBERG et al., 2011), calibração do equipamento (HEYMSFIELD et al., 2018; KOUCHI, 2012) e suporte estrutural (SCHWARZ -MUELLER; MARSHALL; SUMMERSKILL, 2018) para manter todo o equipamento em posições fixas ou em movimento conjunto. Em trabalhos anteriores (BARBIERI et al 2018; SIERRA et al., 2018) foi desenvolvida uma plataforma de escaneamento que pode ser utilizada com diversos escâneres e que foi adotada neste estudo para tentar resolver alguns dos problemas de fixação e movimentação simultânea dos escâneres.

Quanto à qualidade dimensional da malha, é importante considerar qual será o uso final da antropometria digital, porque, ao modelar roupas e outros produtos de consumo, algumas discrepâncias de tamanho podem ser aceitáveis, mas não ao considerar aplicações médicas (VERWULGEN et al., 2018; GUIDI ; GONIZZI; MICOLI, 2016).

Resultados conflitantes têm surgido em relação à qualidade obtida com o Kinect, com alguns trabalhos propondo que é possível usá-lo em situações para escaneamento de corpo inteiro e não para pequenas partes do corpo (VITALI; RIZZI, 2018) também, alguns apenas o endossam para a indústria do vestuário (BRAGANÇA et al., 2018; VITALI; RIZZI, 2018; SOILEAU et al., 2016). Da mesma forma, alguns trabalhos encontraram subestimação de medidas (ALAVANI; KAMAT, 2015) e algumas superestimações (CLARKSON et al., 2014), no geral, encontraram erros inferiores a $0,5 \mathrm{~mm}$.
Pode-se considerar que, conhecendo essa limitação, o equipamento pode ser usado para situações bem pensadas e planejadas. Além disso, é fundamental notar que o aspecto de baixo custo é benéfico para estudos que apresentam restrições de custo (GLEASON et al., 2018; TARABINI et al., 2018; VITALI; RIZZI, 2018).

A outra variável neste estudo é a capacidade do sujeito de manter a posição pelo tempo fixo necessário (SCHWARZ-MUELLER; MARSHALL; SUMMERSKILL, 2018). Mesmo para jovens adultos com capacidades no corpo inteiro, manter uma postura por mais de 5 minutos é doloroso e desconfortável (KEE; KARWOWSKI, 2001). Ao considerar a possível participação de idosos, crianças e pessoas com deficiência, isso é ainda mais presente (BUSSELL; MICHAUD, 1981). Se somar-se à isso o fato de que grande parte dos estudos de antropometria digital tendem a deslocar (girar) o participante (CUI; STRICKER, 2011) em vez do equipamento, a capacidade de manter a postura diminui porque está se adicionando equilíbrio à situação (SCHWARZ-MUELLER; MARSHALL; SUMMERSKILL, 2018). Neste estudo, eliminou-se esse fator desenvolvendo uma plataforma que mantém os sujeitos estáveis e movese os sensores ao seu redor (BARBIERI et al 2018; SIERRA et al., 2018).

Outro ponto em relação ao tempo é que pode haver uma relação entre o tempo de escaneamento e a qualidade da malha. Tempos de escaneamento mais curtos tendem a deixar buracos e espaços em branco que aumentam potencialmente o tempo de pósprocessamento do modelo digital (HEYMSFIELD et al., 2018; SHU et al., 2013). O tempo de escaneamento ideal é aquele que os participantes se sintam à vontade o suficiente para manter a postura pelo tempo determinado e que a digitalização seja o mais completa possível.

Sobre a posição adotada pelos participantes, a ISO 7250 (2017) propõe algumas posturas que podem ser usadas para fazer as medições digitais. No entanto, essas posturas nem sempre são compatíveis com a digitalização em 3D, pois quando os braços são posicionados encostados ao lado do corpo, eles são unidos ao corpo na malha digital, desafiando a coleta de medidas. A ISO 20685-1 (2018) tenta resolver isso, mas, ao fazê-lo, elimina a possibilidade de coleta de algumas das medidas. Algumas outras posições também alteram a postura do corpo inteiro (SCHWARZ-MUELLER; 


\section{MARSHALL; SUMMERSKILL, 2018) e o} tamanho das partes do corpo (CHOI; ASHDOWN, 2011) e, portanto, elas precisam ser selecionadas com cuidado. Finalmente, algumas posturas são mais desconfortáveis que outras (KEE;

KARWOWSKI, 2001), porque solicitam luta contra a gravidade e só podem ser mantidas por períodos mais curtos; outras ainda são impossíveis de manter, dependendo da força física, patologias e deficiências dos participantes.

Considerando esse contexto, admite-se a hipótese de que, ao utilizar escâneres de baixo custo, as principais influências na qualidade da malha são as posturas adotadas pelos participantes, o tempo de escaneamento e seu conforto em relação a postura que afetam sua capacidade de manter a postura.

Para testar esta hipótese, desenvolveu-se um experimento para determinar as melhores posturas, considerando as normas, as limitações do equipamento de baixo custo, a qualidade da malha obtida, o tempo necessário para realizar as digitalizações, as análises ergonômicas das posturas, o conforto do participante e nosso hardware de digitalização. O objetivo final foi gerar recomendações para aprimorar protocolos para estudos antropométricos em larga escala.

Cabe ressaltar que esta é uma versão ampliada e revisada do artigo originalmente publicado nos anais do $17^{\circ}$ Congresso Internacional de Ergonomia e Usabilidade de Interfaces Humano Tecnologia denominado Avaliação de posturas para antropometria digital considerando tempo, conforto e qualidade da malha (SIERRA et al., 2019), publicado à convite da organização deste periódico e do evento.

\section{Método}

Este experimento foi desenvolvido para avaliar a influência de diferentes posturas em termos da capacidade de manter a postura pelo tempo determinado, conforto, ergonomia e qualidade da malha obtida por meio de escaneamento tridimensional, de maneira reconhecer e discutir os principais fatores que afetam a qualidade da malha obtida e consequentemente a qualidade da antropometria digital que pode ser realizada usando a malha obtida.

O experimento foi realizado utilizando uma plataforma estática com um braço giratório que comporta 3 Kinect 360 (MICROSOFT, 2019) fixados por meio de suportes próprios fabricados digitalmente e que tem giro livre controlado pelos pesquisadores. Para o experimento foi utilizado um giro completo de $360^{\circ}$ na orientação horária ou dois giros completos totalizando $720^{\circ}$ sendo o primeiro na orientação horária e a segundo na anti-horária.

As posturas escolhidas foram 6 posições de braço mescladas em 3 posturas da ISO (2018), ISO (2017) e Spahiu, Shehi e Piperi (2016), e podem ser vistas na Figura 1. As posições das pernas foram as mesmas com as pernas levemente entreabertas com os pés completamente apoiados na base da plataforma. As restrições de tempo foram impostas pelo tempo que levou para a plataforma ser girada uma ou duas vezes.

Figura 1: Posturas realizadas no experimento.

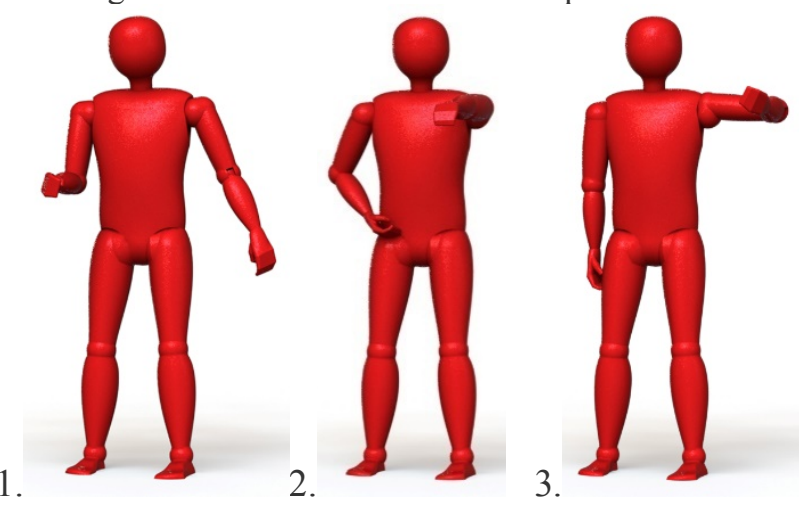

Fonte: Os autores (2019).

Para a análise do conforto, utilizou-se a Escala de Conforto Geral de Shackel (SHACKEL; CHIDSEY; SHIPLEY, 1969), que é uma escala de classificação de onze pontos onde quanto maior a nota dada maior o nível de desconforto. A escala é a seguinte: 1. Me sinto completamente relaxado; 2 . Me sinto perfeitamente confortável; 3 . Me sinto bastante confortável; 4. Me sinto pouco confortável; 5 . Me sinto desconfortável; 6. Me sinto inquieto; 7. Me sinto tenso; 8 . Me sinto rígido; 9 . Me sinto entorpecido; 10. Me sinto dolorido e sensível; 11. Sinto uma dor insuportável.

A avaliação ergonômica da postura foi feita usando as ferramentas de análise ergonômica REBA Rapid Whole Body Analysis ou análise rápida de corpo inteiro (HIGNETT; MCATAMNEY, 1997) e RULA Rapid Upper Limb Analysis ou análise rápida de membros superiores (MCATAMNEY; CORLETT, 1993). 
O REBA fornece um número de 1 a 11 , que reflete o nível de risco para as posturas completas avaliadas. Eles são divididos em cinco da seguinte forma: 1 risco insignificante; entre e 2 e 3 - baixo risco; entre 4 e 7 - risco médio; entre 8 e 10 - alto risco, deve ser modificado; e 11 - risco muito alto, as mudanças precisam ser implementadas imediatamente. A análise REBA foi feita considerando que o participante ocupava as posições demonstradas na Figura 1 por 3 minutos sem movimentação, vibração ou adição de peso e foi considerado o membro superior que dava maior pontuação de risco.

Para a análise das posturas individuais dos braços, visto que são os elementos posturais que se alteram no experimento, foi utilizado o RULA, que, similar ao REBA, fornece notas para avaliação do risco das posições dos membros superiores. As notas do RULA variam entre 1 e 7 , sendo que notas 1 e 2 indicam postura aceitável, 3 e 4, possibilidade de requerer mudanças, 5 e 6 , necessidade de realizar mudanças e 7, necessidade de mudanças imediatas. O RULA fornece notas para os dois lados do corpo separadamente o que é ideal para a avaliação das posturas dos braços utilizadas no experimento.

A qualidade da malha foi analisada seguindo o MAP - Mesh Analysis Protocol, desenvolvido pelos autores, que fornece uma nota numérica para a malha obtida de 0 - inutilizável à 100 - ideal e considera que uma malha utilizável tem uma classificação entre 40 e 100 e que uma ótima malha é de 80 a 100. Ele avalia erros de calibração, erros de costura (desacordo entre o início e o final da malha), furos, objetos estranhos, movimento durante a digitalização, limitações de software e hardware, saliências e erros de reconstrução.

\subsection{Configuração experimental}

O experimento realizado foi o seguinte: primeiro, os participantes receberam as tarefas e foram solicitados a assinar termos de consentimento. Após assinarem, foram digitalizados três vezes, uma em cada postura, em ordem aleatória. O participante manteve-se estático na plataforma e os escâneres foram girados no seu entorno manualmente. A ordem das posturas e o número de voltas foram randomizados para evitar vieses como cansaço e aprendizado. Para cada escaneamento foi cronometrado o tempo de duração.

Após cada escaneamento, os participantes foram solicitados a responder ao nível de escala de conforto (SHACKEL; CHIDSEY; SHIPLEY, 1969) referente à postura realizada. Em seguida, classificou-se cada escaneamento usando o protocolo MAP e tabulados os dados de duração de escaneamento e notas da escala de conforto (SHACKEL; CHIDSEY; SHIPLEY, 1969).

Na Figura 2, é apresentada uma visão geral da configuração do experimento, que também contém uma ilustração da plataforma de escaneamento.

Figura 2: Configuração experimental.

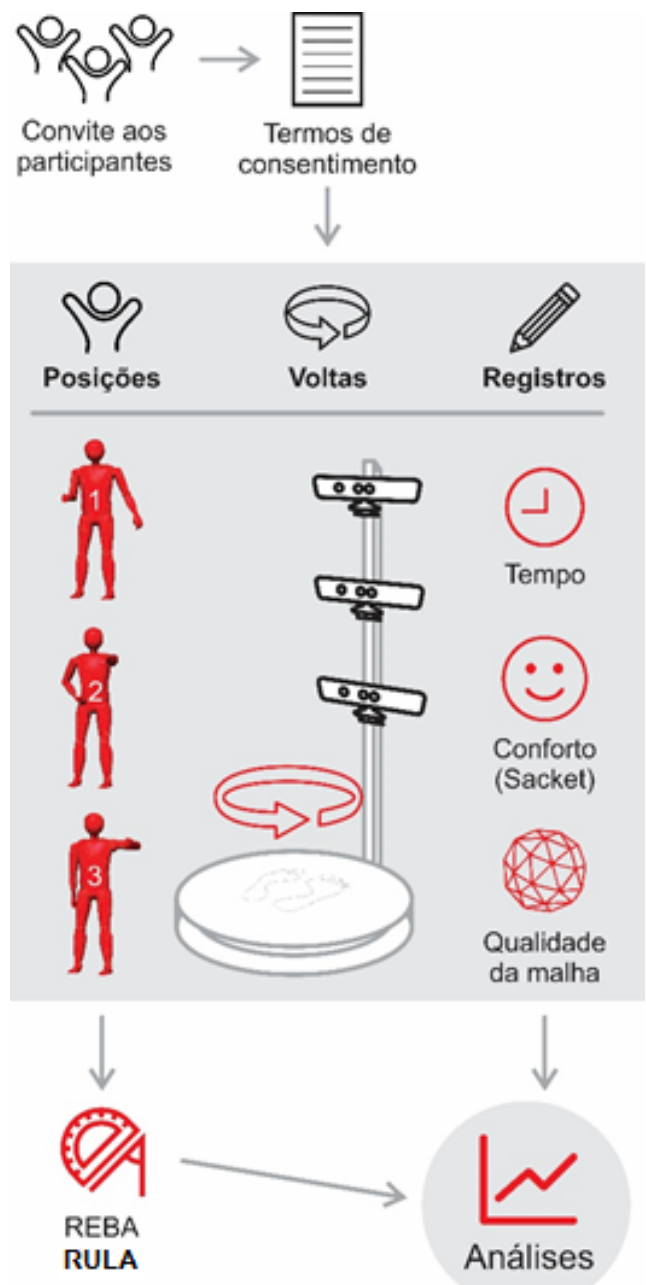

Fonte: Os autores (2019).

Simultaneamente ao experimento também foi feita a análise ergonômica e biomecânica das posturas utilizando as ferramentas REBA (HIGNETT; MCATAMNEY, 1997) e RULA (MCATAMNEY; CORLETT, 1993), já descritas.

Como o objetivo era analisar as posturas e a própria configuração do experimento, não foi realizado nenhuma redigitalização, mesmo que houvesse problemas com a postura adotada pelos participantes 
ou com os escâneres, para permitir a discussão sobre a compreensão das posturas e capturar as limitações do sistema de escaneamento.

\subsection{Materiais}

A plataforma de digitalização usada funciona com 3 Kinects 360 (MICROSOFT, 2019). A plataforma é construída em barras de metal e superfícies de madeira com suportes impressos em 3D para os Kinect 360 que podem ser ajustados em altura manualmente. O raio da plataforma é de um metro.

O software de digitalização utilizado foi o RecFusion (RECFUSION, 2019) e o limpador de malha foi o Meshmixer (AUTODESK, 2019). Para o funcionamento dos Kinect 360, foi utilizado um laptop apropriado com alto poder de processamento e, para o registro dos participantes e dos dados para análise, outro laptop padrão.

No experimento, estiveram presentes cinco pesquisadores que realizaram as diferentes tarefas de registro dos participantes, controle de escaneamento, registro de dados e manipulação da plataforma.

\subsection{Seleção da amostra}

A estratégia de amostragem utilizada foi a de amostra por conveniência, pois o objetivo era comparar e escolher posturas de escaneamento e não fazer inferências estatísticas. Da amostra obtida por conveniência, apenas foram convidados a participar do experimento adultos saudáveis e sem queixas físicas, doenças ou problemas osteomusculares no momento do experimento.

\section{Resultados}

A seguir, são apresentados os dados para caracterização da amostra, qualidade da malha obtida com o MAP, conforto do participante (SHACKEL; CHIDSEY; SHIPLEY, 1969), análise da postura com REBA (HIGNETT;

MCATAMNEY, 1997) e RULA (MCATAMNEY; CORLETT, 1993), e tempos de escaneamento. Em continuidade são discutidas as relações entre postura, tempo, qualidade da malha e conforto; e são feitas recomendações finais sobre as posturas e recomendações para o protocolo de escaneamento.

Nos resultados os sujeitos participantes são representados por 'S' (S1-S16), as posturas por 'P'
(P1, P2, P3 na ordem apresentada na Figura 1) e o número de voltas por V1 (uma volta) e V2 (duas voltas).

\subsection{Caracterização da amostra}

A amostra foi composta por dezesseis participantes (6 mulheres e 10 homens) (Tabela 1) componentes da amostra de conveniência. Todos assinaram termos de consentimento para participar do experimento. Suas idades variaram de 24 a 60 anos e eram todas saudáveis, sem restrições médicas que dificultavam sua capacidade de manter as posturas solicitadas.

Tabela 1: Caracterização da amostra.

\begin{tabular}{|c|c|c|}
\hline & Mulheres $(\mathbf{n = 6})$ & Homens $(\mathbf{n = 1 0})$ \\
\hline Altura (cm) & $159,8 \pm 4,9$ & $177,4 \pm 6,1$ \\
\hline Peso (kg) & $69,3 \pm 12,6$ & $82,2 \pm 10,7$ \\
\hline \multicolumn{3}{|c}{ Fonte: Os autores (2019). }
\end{tabular}

No total, foram feitas 48 digitalizações (três para cada participante).

\subsection{Qualidade da malha}

Utilizando o protocolo MAP, foi possível classificar todas as malhas obtidas nos seguintes erros: calibração, erros de costura, buracos, objetos estranhos, movimento durante a digitalização, limitações de software e hardware, saliências e erros de reconstrução. Em seguida a descrição desses erros encontrados.

Os erros de calibração acontecem com a movimentação não simultânea de um ou mais escâneres no momento da digitalização causando desvio de parte da malha. Os erros de costura são erros que acontecem no encontro entre o início da digitalização e seu fim, sendo parte natural do processo de escaneamento. Os orifícios acontecem por causa de sombras ou geometrias complexas que dificultam o acesso do infravermelho à superfície causando buracos na malha.

Os objetos estranhos são causados pela digitalização de elementos no entorno do que está sendo escaneado e não estão ligados à malha principal. Quando esses elementos estranhos estão ligados à malha são chamados de saliências.

A movimentação dos participantes durante o escaneamento também causa erros como buracos ou alterações de geometrias e finalmente as limitações de software e hardware podem causar erros como 
erros de reconstrução com a simplificação ou adição de rugosidades à malha, e cortes abruptos de elementos sendo escaneados e até mesmo incapacidade de gerar uma malha.

As pontuações finais do MAP (Gráfico 1), que avalia esses erros comentados, para o experimento variaram de 38,1 a 89,5 , permanecendo acima da linha recomendada, exceto o S6 com duas voltas que teve nota 38,1 e pode ser considerada inutilizável. Isso aconteceu pela movimentação involuntária excessiva do participante dado seu desconforto na posição requerida.

Gráfico 1: Notas MAP para diferentes posturas.

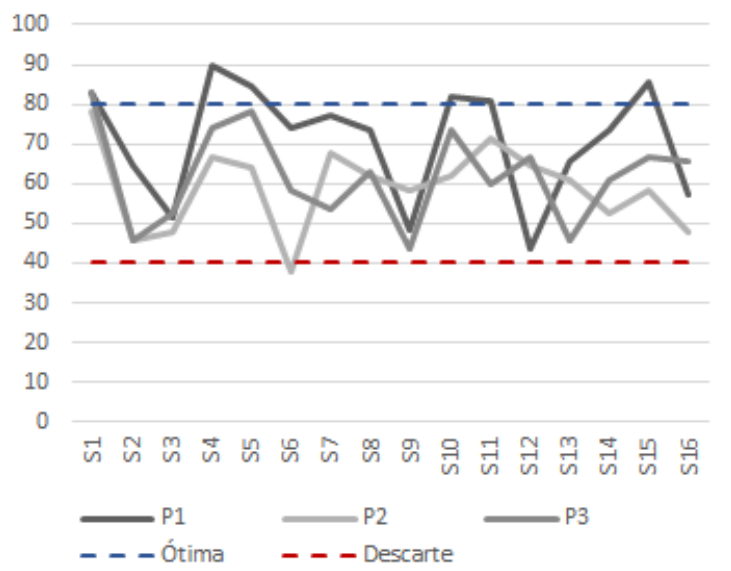

Fonte: Os autores (2019).

Em média, a primeira postura (P1) gerou as melhores malhas com algumas excedendo a linha de malha "ótima" e a segunda postura (P2) foi a pior sendo a única a gerar uma malha inutilizável.

Dos erros encontrados, alguns eram esperados porque não foram limitadas as roupas usadas pelos participantes e algumas dobras de roupa alteram a qualidade das digitalizações pois são geometrias complexas que geram buracos. Na Figura 3 são apresentados alguns dos erros gerados causados pelas limitações do experimento.
Figura 3: Erros de limitação do experimento.

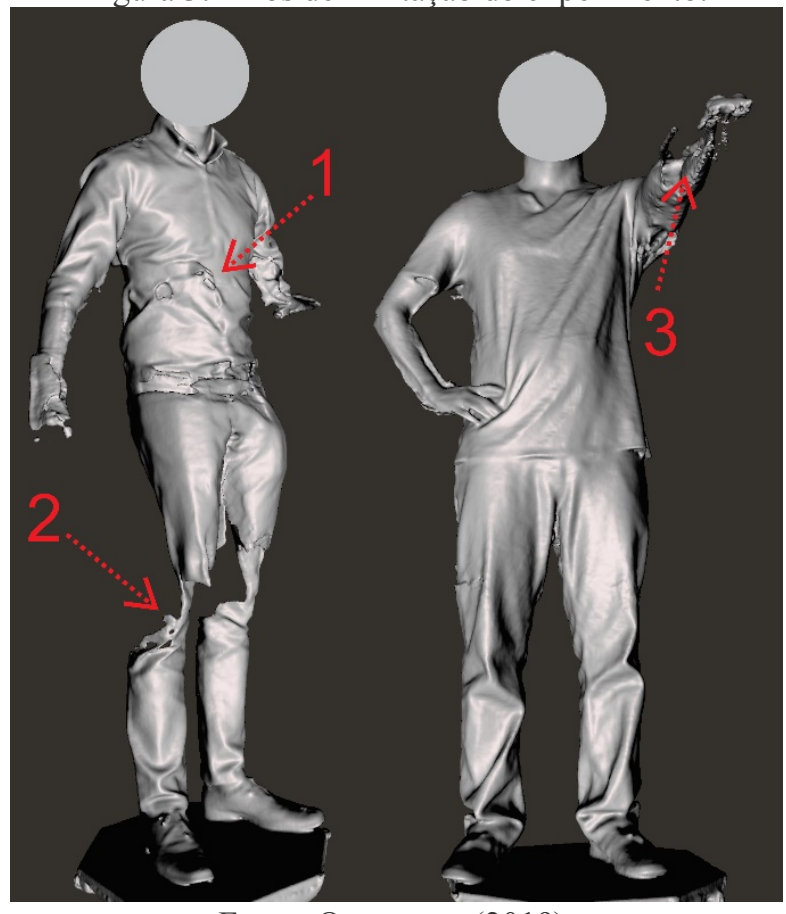

Fonte: Os autores (2019).

O primeiro erro, já comentado (Figura 3 - 1) é causado pelas roupas utilizadas pelos participantes. Isso reafirma a necessidade de utilização de roupas ajustadas ao corpo ou sem roupas para o escaneamento o que implica também na realização do escaneamento em ambiente privado.

O segundo erro, de limitação do equipamento (Figura 3 - 2) foi a descalibração dos Kinect causada pelo desalinhamento de um ou mais Kinect no momento do escaneamento. Esse problema só pode ser verificado após o processamento do escaneamento que gera a malha. Dessa maneira outra indicação para o protocolo é o mantimento do participante no ambiente de escaneamento até o final do processamento da malha para verificação de necessidade ou não de realizar novo escaneamento. O terceiro erro (Figura 3 - 3) é causado pela limitação de distância entre o equipamento e o participante causando perdas na geometria do braço estendidos pois estava muito próximo ao escâner.

Além dos erros de limitação, percebe-se que alguns erros posturais contribuíram para a má qualidade da malha (Figura 4 - 1) e para a má qualidade da postura (Figura 4 - 2) que afeta a antropometria digital com a torção demasiada do corpo. 


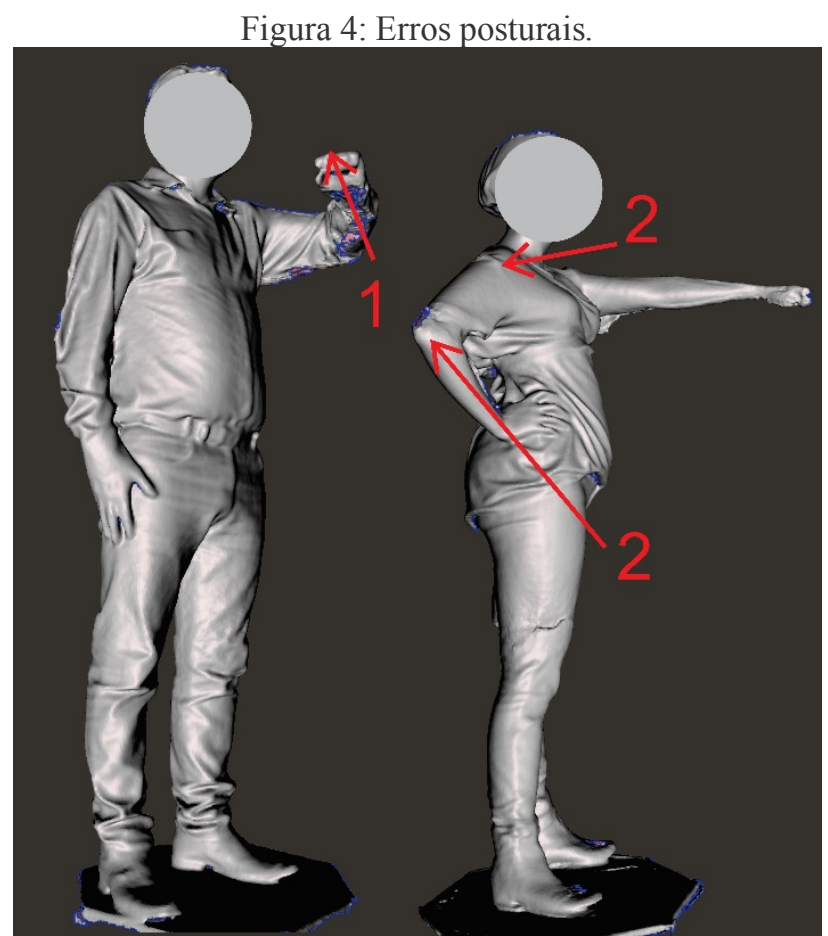

Fonte: Os autores (2019).

A principal postura que afetou a possibilidade de antropometria foi a com as mãos na cintura (Figura 4 - 2) que modificou a posição do corpo inteiro.

Além das notas das malhas, cruzaram-se os dados de nota das malhas e número de voltas para identificar a influência das voltas para a qualidade da malha (Tabela 2).

Tabela 2: Médias das notas MAP para diferentes posturas e número de voltas.

\begin{tabular}{|c|c|c|c|}
\hline & T1 & T2 & $\overline{\mathbf{x}}$ \\
\hline P1 & 71,9 & 69,6 & $\mathbf{7 0 , 7}$ \\
\hline P2 & 59,4 & 58,7 & $\mathbf{5 9 , 0}$ \\
\hline P3 & 56,7 & 67,1 & $\mathbf{6 1 , 9}$ \\
\hline$\overline{\mathbf{x}}$ & $\mathbf{6 2 , 7}$ & $\mathbf{6 5 , 1}$ & \\
\hline
\end{tabular}

Fonte: Os autores (2019).

Como se vê, as posturas adotadas influenciaram mais a qualidade da malha do que o número de voltas que deram resultados semelhantes no MAP.

\subsection{Conforto dos participantes}

Para o conforto dos participantes, as médias foram semelhantes (Tabela 3), permanecendo, no meio da escala de conforto (SHACKEL; CHIDSEY;

SHIPLEY, 1969), nesse caso, a menor pontuação é preferível. As notas médias foram: 4. Sinto-me pouco à vontade; 5 . Sinto-me desconfortável; 6 . Sinto-me inquieto.
Tabela 3: Notas de conforto.

\begin{tabular}{|c|c|c|c|c|c|c|}
\hline \multirow{2}{*}{ Conforto } & \multicolumn{2}{|c|}{ Uma volta } & \multicolumn{2}{|c|}{ Duas voltas } \\
\hline & P1 & P2 & P3 & P1 & P2 & P3 \\
\hline S1 & 5 & & 10 & & 9 & \\
\hline S2 & 4 & & 5 & & 5 & \\
\hline S3 & & 4 & 5 & 4 & & \\
\hline S4 & & 9 & & 6 & & 10 \\
\hline S5 & 4 & 5 & & & & 5 \\
\hline S6 & 1 & & & & 5 & 5 \\
\hline S7 & 4 & & 5 & & 5 & \\
\hline S8 & & & 5 & 4 & 5 & \\
\hline S9 & & 3 & 5 & 3 & & \\
\hline S10 & & 10 & & 5 & & 10 \\
\hline S11 & 3 & 6 & & & & 7 \\
\hline S12 & 4 & & & & 9 & 5 \\
\hline S13 & 2 & & 4 & & 8 & \\
\hline S14 & 4 & & & & 6 & 6 \\
\hline S15 & & 9 & 5 & 3 & & \\
\hline S16 & & 3 & & 6 & & 4 \\
\hline $\bar{x}$ & 3,5 & 6 & 5,5 & 4,5 & 6,5 & 6,5 \\
\hline
\end{tabular}

Fonte: Os autores (2019).

A postura mais confortável foi a postura 1 e a postura menos confortável 2. Os participantes S1, S4 e S10 foram responsáveis pelas notas mais altas, aparentemente independentes da postura ou do número de voltas que leva a acreditar que já estavam de alguma forma com dor ou desconforto muscular antes da digitalização.

O número de voltas, como previsto, afetou o conforto dos participantes pois quanto mais voltas realizadas maior o tempo que precisam manter a posição. No entanto essa alteração não foi significativa e pode ser casual.

\subsection{Avaliação ergonômica}

A análise da postura completa dos participantes, feita utilizando o REBA (HIGNETT;

MCATAMNEY, 1997). Diversas posições corporais se mantiveram para todas as posições, essas características recorrentes foram: posições de pescoço e tronco neutras à $0^{\circ}$ sem torção ou pensão lateral, as pernas estendidas com peso igualmente distribuído e os pés completamente apoiados no chão. Além disso nenhuma posição prevê a adição de peso ou carregamento de ferramentas e todas as posturas foram avaliadas com a sustentação das posições por mais de um minuto.

Para diferenças entre as posições de braços foi considerada a pior possível pontuação entre as posições de braço. Por isso não ser o ideal, em seguida foi realizado o RULA para que possa ser avaliado corretamente as posições dos dois braços separadamente. Para a primeira posição considerou- 
se que os braços estavam abduzidos lateralmente e o braço à $90^{\circ}$ do antebraço com pulsos neutros. Para a segunda posição considerou-se o braço entendido à frente com pulso torcido para apoio na cintura. E para a terceira posição considerou-se o braço abduzido lateralmente à, dobrado à $90^{\circ}$ e ombros levantados. As notas REBA finais para as posturas podem ser verificadas na Tabela 4.

Tabela 4. Notas REBA para as posturas.

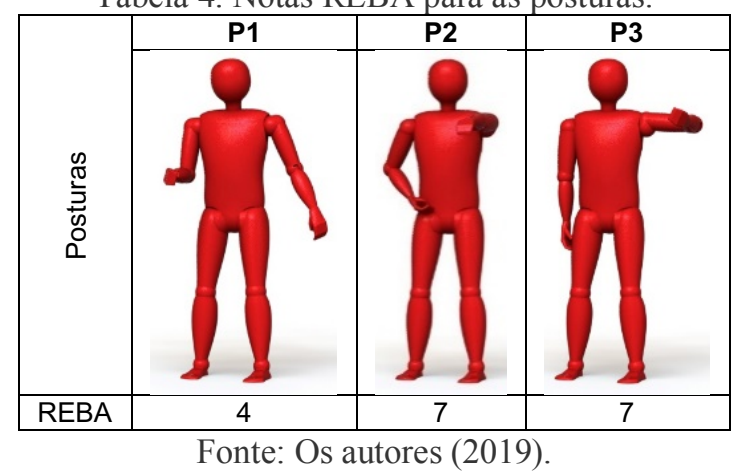

Todas as posturas foram consideradas de médio risco, as pontuações estão relacionadas ao tempo de mantimento estático da postura e extensão e sustentação dos membros o que fez com que apesar da falta de vibrações e pesos e da posição neutra das pernas e a postura ereta do tronco as posturas ainda fossem consideradas de médio risco.

A principal preocupação ergonômica é a posição dos braços estendidos na frente e na lateral do corpo. Por esse motivo optou-se por realizar a análise de risco das posições dos braços individualmente para identificar quais posições eram mais críticas. Essa análise foi feita utilizando o RULA. Apesar dos altos resultados obtidos com o REBA a avaliação individual das posturas dos braços (Tabela 5) indica que as posturas podem ser mantidas, mas as duas últimas que devem ser investigadas.

Tabela 5. Notas RULA para as posturas dos braços.

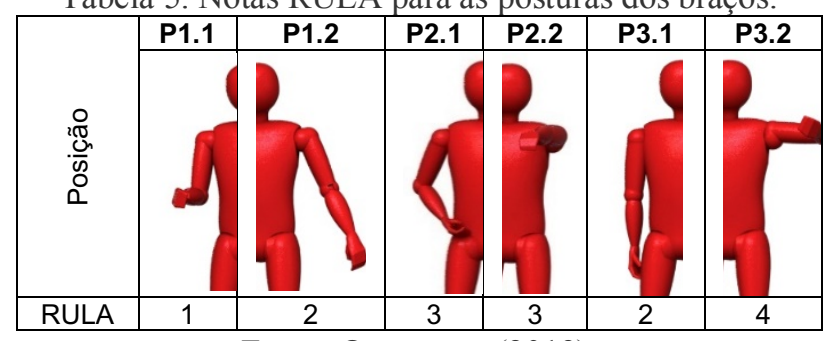

Fonte: Os autores (2019).

A principal diferença entre as ferramentas é que a primeira considera que posturas estáticas são aquelas mantidas por mais de um minuto e a segunda por mais de dez minutos.

\subsection{Tempo de escaneamento}

As diferenças de tempo entre os escaneamentos realizados com uma volta e com duas voltas (tabela 6) não foram proporcionais e tiveram uma média de 66s para uma volta e 94 s para duas voltas.

Tabela 6. Tempo médio para um e dois turnos.

\begin{tabular}{|c|c|c|c|c|}
\hline & P1 & P2 & P3 & $\overline{\mathbf{x}}$ \\
\hline T1 & 67,5 & 65,8 & 66,7 & 66,7 \\
\hline T2 & 93,8 & 97,4 & 91,9 & 94,4 \\
\hline \multicolumn{6}{|c|}{ Os autores (2019). }
\end{tabular}

Essa diferença pode ter ocorrido porque a primeira volta é o responsável por coletar a geometria e a segunda é para suavizar a malha e para a coleta de faces que podem ter sido perdidas na primeira volta e, como tal, é um processo mais rápido.

Devido ao movimento involuntário dos participantes, na segunda volta, observou-se que alguns dos erros de postura se agravaram, deixando a malha com mais erros do que apenas com uma volta que gerou buracos, facilmente corrigíveis, mas não falhas incorrigíveis na malha como as de movimentação e erro no posicionamento.

Também se notou que, como para cada participante foram realizadas três digitalizações, mesmo havendo descanso de alguns minutos entre as digitalizações, a sua sensação de tempo foi prejudicada fazendo que para a terceira digitalização estivessem mais impacientes e fadigados e realizassem mais movimentações involuntárias.

\section{Discussão}

Antes de delinear a postura preferencial e sugestões para o protocolo de escaneamento considerando o conforto dos participantes e qualidade da malha obtida, há algumas reflexões a serem feitas sobre os resultados. Essas reflexões são relativas às relações entre postura, tempo de escaneamento, qualidade da malha e conforto.

A motivação para a realização desse experimento se deu pela dificuldade em identificar quais são os principais fatores humanos que afetam os resultados do escaneamento com foco em antropometria digital avaliado de acordo com a qualidade da malha gerada. Considerações acerca da qualidade dos escâneres, softwares de escaneamento e precisão dos escâneres são recorrentes (BRAGANÇA et al., 2018; GLEASON et al., 2018; HEYMSFIELD et 
al., 2018; BRAGANÇA et al., 2017; BRENDLER et al., 2016; GUIDI; GONIZZI; MICOLI, 2016; etc.) no entanto, os efeitos dos fatores humanos para esses resultados geralmente não são reconhecidos.

À princípio selecionaram-se participantes da população em geral, saudáveis e sem doenças ou deficiências que afetariam a sua capacidade em participar do experimento, assim como seria realizado em um grande estudo antropométrico populacional. No entanto, encontrou-se que alguns participantes não conseguiram participar efetivamente do estudo com reclamações de dores, desconforto, dificuldade em atingir algumas posições e impaciência em relação ao tempo total de participação, que durou entre 10 e 15 minutos.

Esse é o primeiro fator humano a ser considerado. A livre declaração de condições físicas nem sempre é refletida na realidade podendo haver omissões e até mesmo desconhecimento de condições físicas de incapacidade. Para tanto, caso a configuração experimental pretendida exija a adoção de posturas diferenciadas é necessário realizar um treinamento prévio com os participantes ou até mesmo avaliação física antes da realização do escaneamento.

Outro fator humano presente antes mesmo da realização do escaneamento é a sua privacidade, tanto para a troca de roupas, quanto para a realização do escaneamento que é feito em presença de diversos pesquisadores pois há necessidade do controle de equipamentos tecnológicos que não podem ser controlados por apenas uma pessoa. Sendo assim, o participante deve estar consciente concordante de que este será o caso.

Ainda, esse é um fator incontornável pois as roupas são os principais elementos que afetam a qualidade da malha obtida e a acurácia dimensional das medidas a serem retiradas da malha. Idealmente o escaneamento seria realizado sem o uso de roupas ou com roupas perfeitamente ajustadas ao corpo sem dobras ou deformações.

Sobre as posições selecionadas, de maneira a simplificar e aligeirar o experimento optou-se pela utilização de duas posições de braço diferentes para cada postura, sendo uma com o braço estendido e outra com o braço dobrado. No entanto esse caso não foi ideal pois causou alteração postural da coluna dos participantes o que afetaria a qualidade final das medidas.
Ainda assim como neste experimento o objetivo não era a efetiva realização das medidas e sim a verificação da qualidade da malha obtida com o escaneamento e como era afetada pelas posturas, conforto e tempo, a configuração experimental concebida foi suficiente.

Foram usadas cinco ferramentas de avaliação para o estudo, escala de conforto (SHACKEL; CHIDSEY; SHIPLEY, 1969), protocolo de avaliação da malha MAP, e medição de tempo, REBA (HIGNETT; MCATAMNEY, 1997) e RULA (MCATAMNEY; CORLETT, 1993).

A escala de conforto, apesar de ser subjetiva, forneceu dados importantes para identificação da relação entre postura selecionada e capacidade de manter a postura. Para os resultados de conforto houve grande discrepância para as notas da primeira postura $(3,5$ e 4,5$)$ e para as da segunda e terceira que foram similares entre si (6 e 6,5). Indicando problemas nas duas últimas posturas.

O mesmo padrão foi traduzido com a nota de qualidade da malha onde pôde ser identificada a superioridade da qualidade gerada pela primeira postura em relação às outras. Para a primeira postura 6 malhas obtiveram notas que indicam qualidade ótima da malha. Nenhuma outra postura obteve alguma malha com nota ótima.

A marcação do tempo de escaneamento demonstra outra questão relativa aos fatores humanos, nesse caso voltado aos técnicos realizando a digitalização. A plataforma é de controle manual, e a limitação de velocidade do giro da plataforma é determinada pela pessoa que está realizando o escaneamento de acordo com os resultados automaticamente apresentados na tela do computador. No entanto, os técnicos não são exatos nessa atividade, resultando em casos com maior tempo de escaneamento e casos com menor. E, ainda mais, com tempos não proporcionais para uma ou duas voltas da plataforma. Na segunda volta, como já foi realizada a maior parte da coleta, é possível realizar o giro com maior velocidade, otimizando o tempo de escaneamento, no entanto esse tipo de conhecimento depende da formação e competência dos pesquisadores e técnicos.

Para uma situação mais padronizada seria recomendado utilizar uma plataforma com giro automatizado para equivaler os tempos de escaneamento. Mas, isso pode causar outros 
problemas pois geometrias mais complexas são de maior lentidão de tempo de escaneamento o que pode casar perda de algumas geometrias com a automatização do processo.

As ferramentas de análise ergonômica REBA (HIGNETT; MCATAMNEY, 1997) e RULA (MCATAMNEY; CORLETT, 1993), puderam ser utilizadas para identificar que, provavelmente, as dificuldades encontradas pelos participantes em atingir algumas das posições, notadamente as posições 2 e 3 , tinham fundamentação no desconforto musculoesquelético, sendo que são posturas utilizáveis mas que não devem ser realizadas por longos períodos de tempo e nem mesmo diversas vezes pois são potenciais causadoras de danos.

Sobre os resultados, parece haver uma maior influência da postura na qualidade da malha do que do tempo na qualidade da malha. Embora o tempo de duas voltas da plataforma tenha sido $40 \%$ maior, o aumento na qualidade da malha não justificou o período prolongado, melhorando apenas 2,5 pontos (de uma escala de 100 pontos). Isso leva a crer que os problemas de malha estavam relacionados às limitações do equipamento e à postura selecionada indiferentemente do tempo em que o participante manteve a postura.

A primeira postura, com um dos braços levemente suspenso e o outro dobrado na posição padrão de medição antropométrica manual, apresentou a melhor qualidade de malha, primeiro porque não tocava o corpo e não soldava a área e, segundo, por ser uma postura mais fácil para manter pelo período especificado. A pior postura, que foi a segunda, com um dos braços estendidos e o outro dobrado na cintura, apresentou a pior qualidade de malha, devido à dificuldade de manter o braço estendido na frente do corpo, fazendo-o balançar e dificultando a fechamento da malha. Além disso, para pessoas com comprimento de braço maior que o tamanho máximo da plataforma, parte dos braços e da cabeça, não foram possíveis de digitalizar deixando um buraco aberto.

Acredita-se que, se uma postura exata é necessária para o experimento, os pesquisadores devem orientar o usuário a atingir a postura adequada, produzindo pistas visuais para ele seguir, adicionando guias, figuras ou espelhos para que os participantes possam se controlar e corrigir sua postura automaticamente. $\mathrm{O}$ espelho deve ser colocado diretamente na frente dos sujeitos, para que eles não precisem mudar a posição da cabeça para se olhar.

O outro problema associado com a qualidade da malha foi a calibração que dificultou a qualidade de algumas digitalizações. Isso poderia ter sido corrigido na época, mas optou-se por não o fazer para demonstrar que isso pode ocorrer. Para estudos antropométricos reais, esse problema pode ser evitado ou corrigido com a recalibração dos escâneres de vez em quando. Se o equipamento descalibrar para um participante, sua digitalização pode ser refeita com a calibração correta. Nesse contexto, a pontuação MAP pode ser usada para determinar se a digitalização deve ser refeita ou não, de acordo com a pontuação fornecida.

O conforto do participante, como previsto, influenciou a qualidade das digitalizações. Alguns, mesmo com relatos de não ter condições prévias que os impedissem de manter a posição, tiveram dificuldade e relataram grandes níveis de desconforto. Isso não estava relacionado à idade $\mathrm{e}$ provavelmente foi a causa de algum desconforto musculoesquelético anterior aos exames ou limiar geral de dor mais baixo. Ao extrapolar isso para populações mais específicas, como idosos e crianças, essa é uma questão importante a ser observada. Mesmo que a postura seja tolerável para a maioria dos participantes, alguns podem não ser capazes de mantê-la, e isso pode exigir posições alternativas.

A maioria dos problemas encontrados pode ser corrigida com o uso de roupas adequadas, alterações na plataforma de digitalização, como comprimentos e alturas variáveis para diferentes participantes e alterações no protocolo, especialmente na calibração dos escâneres, se estiver trabalhando com mais de um. Para agilizar o processo para um número maior de participantes, eles podem ser divididos em percentis de altura antes da digitalização e digitalizados em grupos com comprimentos de plataforma apropriados para o tamanho dos participantes.

\subsection{Recomendações sobre posturas e protocolo de digitalização}

Por fim, escolheu-se a melhor postura (Figura 5), que proporcionou a melhor qualidade de malha e foi considerada a mais confortável para os participantes, para que pudessem sustentá-la por mais tempo. A classificação na ordem de melhores 
posturas foi, a primeira como a melhor, em seguida da terceira, seguida pela segunda como postura que gerou mais erros e foi considerada a mais desconfortável.

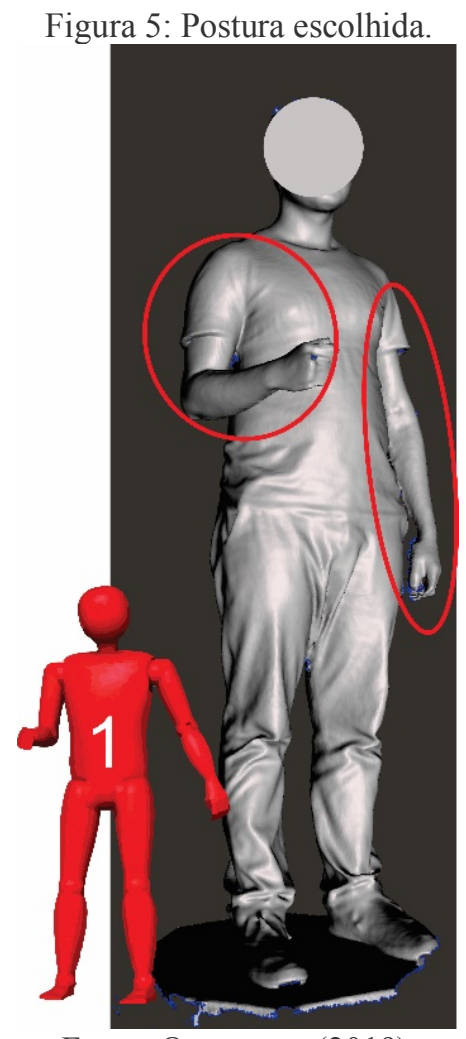

Fonte: Os autores (2019).

Para a primeira postura, apesar de ser a considerada melhor, para alguns casos houve fusão das geometrias pois apesar de haver a recomendação de deixar os braços levemente afastados do corpo a fadiga dos participantes fez com que alguns, principalmente nos casos em que esta era a terceira postura de digitalização, acabassem apoiando os braços ao lado do corpo. Apesar de isso não afetar na qualidade da malha, afeta na capacidade de coleta de medidas do braço e tronco dessa pessoa. Por esse motivo recomenda-se o fornecimento de apoiadores para os braços de maneira a padronizar ainda mais a posição e evitar a fadiga da luta dos braços contra a gravidade.

Na segunda postura (Figura 6) pode-se observar duas questões, a primeira concerne o braço dobrado com as mãos apoiadas no quadril. Assim como no caso da primeira postura, essa posição forneceu uma boa qualidade de malha, no entanto a mão na cintura atrapalhou a coleta de medidas do quadril pois há a fusão entre as geometrias da mão e do quadril. Para a segunda questão, o braço estendido à frente foi a segunda posição mais desconfortável de todas, que além de causar movimentações involuntárias e afetar a postura geral da coluna, em diversos casos foi maior do que a distância mínima do Kinect, havendo perda da coleta de quase toda a geometria dos braços. A postura 2, que é a soma dessas duas posições comentadas, no total causou rotações do tronco dos participantes como demonstrado na Figura 4.

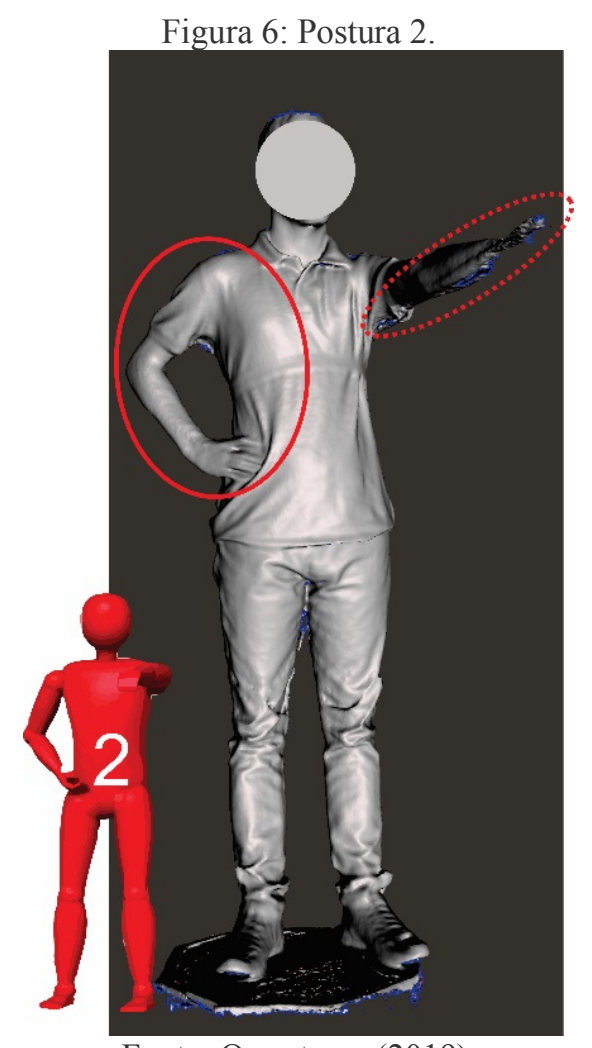

Fonte: Os autores (2019).

A terceira postura (Figura 7), teve problemas na coleta das duas posições de braço. Na posição com o braço estendido ao lado do corpo houve fusão completa entre o braço e o tronco causando dificuldade de realizar medidas tanto dos braços como do tronco. Além disso, gerou geometrias complexas entre essas duas partes, o que causou problemas na malha. A posição do braço abduzido lateralmente e flexionado e foi considerada a pior em termos de risco musculoesquelético e também foi considerada a mais desconfortável, alterou a posição do tronco e causou movimentações involuntárias. No entanto se comparada com o braço estendido da segunda postura, foi melhor para a coleta pois não passou dos limites mínimos de coleta do Kinect, sendo possível, quando o participante conseguiu manter a postura estática, a coleta da geometria de todo o braço. 


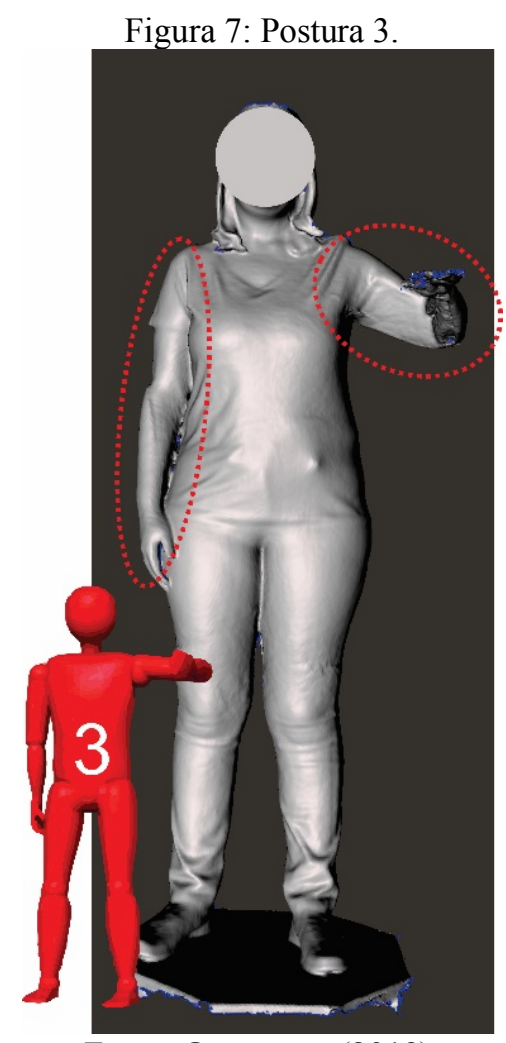

Fonte: Os autores (2019).

Feitas essas avaliações, apesar de ter sido escolhida a primeira postura como a melhor, sugere-se que na coleta haja a garantia dos espaçamentos entre os membros superiores e o tronco, fornecendo apoios e separadores. Ainda se sugere que, se possível, sejam realizadas duas coletas, uma com os participantes com os dois braços estendidos e outra com os dois braços dobrados visto que diferentes posições de braço para cada lado do tronco afetam a postura da coluna causando torções.

Além das posturas, existem outras recomendações necessárias para garantir a qualidade das digitalizações, incluindo menos furos, menos tempo e melhor conforto dos participantes. São elas: fazer apenas uma volta com a plataforma, usar roupas apropriadas, incluir ajustes de altura e comprimento na plataforma para melhor acomodar diferentes percentis, otimização da escaneamento com agrupamento de participantes antes do escaneamento e recalibração do sistema.

A escolha de uma volta é proposta com base nos resultados deste experimento, a segunda volta não aumenta a qualidade da malha e o tempo adicionado afeta a capacidade dos participantes de manter a postura. Além disso, a roupa afeta a digitalização e a coleta de medidas e morfologia. As dobras são furos em potencial e causam geometrias incorretas que precisarão ser limpas posteriormente. As roupas apropriadas são, se possível, roupas mínimas, como roupas íntimas ou roupas de praia e cabelos presos. Se não for possível, as roupas devem ser ajustadas, como roupas de ginástica.

As alterações na plataforma e no agrupamento de participantes são específicas para essa configuração experimental, mas são necessárias para garantir a captura correta de todo o corpo de todos os participantes. O equipamento possui restrições de distância (o sujeito precisa estar entre 50 e $60 \mathrm{~cm}$ do escâner); portanto, se a distância for muito grande, a geometria não será coletada. Se alguns dos participantes são muito pequenos e alguns são altos demais, as alterações de distância podem afetar a capacidade de obter toda a malha e, nesse contexto, a plataforma precisa ser ajustável.

Finalmente, a recomendação de calibração veio da experiência de que a cada cinco ou seis participantes ocorreram alguns erros de calibração. Se os escaneamentos estiverem sendo feitos e nenhum erro dessa natureza ocorrer, essa etapa poderá ser ignorada até que seja necessária. Se a etapa não for executada, pelo menos a cada cinco escaneamentos a malha precisa ser verificada, usando o protocolo MAP, por exemplo, para verificar a necessidade de recalibração.

\section{Considerações finais}

Com esse experimento, descobriu-se que as posturas adotadas tiveram mais influência na qualidade da malha, depois do tempo e do número de voltas para a digitalização. Isso prova que nossa hipótese estava correta, especialmente para o efeito de uma postura exercida sobre a qualidade da malha. $\mathrm{O}$ experimento também ajudou gerar recomendações para melhorar o protocolo de escaneamento, com as principais alterações e posições destacadas no item 4.1.

O conforto, embora seja um assunto subjetivo, foi útil para discutir e escolher entre as posturas.

Mesmo para a amostra pequena, houveram resultados conflitantes que provavelmente também seriam verificados com amostras maiores. As outras medidas, como qualidade, avaliação ergonômica e tempo, também foram eficazes na análise dos dados e facilmente coletadas.

Por causa da variabilidade adicionada ao lidar com pessoas diferentes, e por causa da influência que essas pessoas podem ter na qualidade do estudo 
PUC-Rio Pontifícia Universidade Católica do Rio de Janeiro Departamento de Artes \& Design | PPGDesign

geral, elas sempre devem ser cuidadosamente consideradas. A segurança e o bem-estar dos participantes devem ser uma das primeiras considerações ao planejar um experimento.

Este trabalho, ao responder perguntas sobre as condições do experimento, também pode ser uma diretriz para a preparação e verificação de outras configurações experimentais em relação à participação do sujeito.

A digitalização tridimensional como ferramenta de desenvolvimento de produtos ergonômicos vem surgindo como uma opção viável, segura e rápida, e agora é a hora de considerar suas possibilidades e limitações, especialmente no que diz respeito aos fatores humanos e design centrado no usuário, mesmo no estágio de coleta de dados.

Ao analisar estudos antropométricos e de desenvolvimento de produtos usando escâneres 3D, a postura e as posições são frequentemente ignoradas e pouco descritas. Novos protocolos, considerando os usuários e suas limitações, precisam ser desenvolvidos para tornar o uso dessa tecnologia mais viável e acessível.

\section{Referências Bibliográficas}

ALAVANI, G. K.; KAMAT, V. Human face anthropometric measurements using consumer depth camera. In: NATIONAL CONFERENCE ON COMPUTER VISION, PATTERN RECOGNITION, IMAGE PROCESSING AND GRAPHICS, 5, 2015, Patna. Anais [...]. Patna: IEEE, 2015. p. 1-4.

AUTODESK. Meshmixer 3.5. Disponível em: http://www.meshmixer.com/. Acesso em: 05 set. 2019.

BARBIERI, B.; SIERRA, I.S.; SEGALLA, V.; OKIMOTO, M.L.L.R. Sistema de suporte para escaneamento tridimensional. Depositante: Universidade Federal do Paraná. 2018. Depósito: 24 ago. 2018.

BRAGANÇA, S. et al. Validation study of a Kinect based body imaging system. Work, v. 57, n. 1, p. 921, 2017.

BRAGANÇA, S. et al. A comparison of manual anthropometric measurements with Kinect-based scanned measurements in terms of precision and reliability. Work, v. 59, n. 3, p. 325-339, 2018.

BRENDLER, C. F. et al. Uso da digitalização 3D do corpo humano para desenvolvimento de produtos personalizados: Análise comparativa entre os escâneres Artec EVA e o Kinect. Estudos em Design, v. 24, n. 2, p. 24-43, 2016.

BUSSELL, P.; MICHAUD, L. Seated anthropometry: The problems involved in a largescale survey of disabled and elderly people. Ergonomics, v. 24, n. 11, p. 831-845, 1981.

CHOI, S.; ASHDOWN, S. P. 3D body scan analysis of dimensional change in lower body measurements for active body positions. Textile Research Journal, v. 81, n. 1, p. 81-93, 2011.

CLARKSON, S. et al. Assessing the suitability of the Microsoft Kinect for calculating person specific body segment parameters. In: EUROPEAN CONFERENCE ON COMPUTER VISION, 13, 2014, Zurich. Anais [...]. Zurich: Sringer, 2014. p. 372-385.

COSTA, T. N. et al. Uso da digitalização 3D e da parametrização de medidas antropométricas para produção de moldes personalizados para o vestuário. Educação gráfica, v. 19, n. 2, p. 122$142,2015$.

CUI, Y.; STRICKER, D. 3D body scanning with one Kinect. In: INTERNATIONAL CONFERENCE ON 3D BODY SCANNING TECHNOLOGIES, 2, 2011, Lugano. Anais [...]. Lugano: 3D Body.tech, 2011.

GARSTHAGEN, R. An open source, low-cost, multi camera full-body 3D scanner. In:

INTERNATIONAL CONFERENCE ON 3D BODY SCANNING TECHNOLOGIES, 5, 2014, Lugano. Anais [...]. Lugano: 3D Body.tech, 2014.

GLEASON, J. R. et al. A safe, low-cost, easy-to-use 3D camera platform to assess risk of obstructed labor due to cephalopelvic disproportion. PloS one, v. 13, n. 9, p. e0203865, 2018.

GUIDI, G.; GONIZZI, S.; MICOLI L. 3D capturing performances of low-cost range sensors for massmarket applications. In: INTERNATIONAL ARCHIVES OF THE PHOTOGRAMMETRY, REMOTE SENSING AND SPATIAL 
PUC-Rio Pontifícia Universidade Católica do Rio de Janeiro Departamento de Artes \& Design | PPGDesign

LEUI | Laboratório de Ergodesign e Usabilidade de Interfaces

INFORMATION SCIENCES, 23, 2016, Prague. Anais [...]. Prague: XXIII ISPRS Congress, 2016, p. 33-40.

HEYMSFIELD, S. B. et al. Digital anthropometry: a critical review. European journal of clinical nutrition, v. 72, n. 5, p. 680-687, 2018.

HIRSHBERG, D. A. et al. Evaluating the automated alignment of 3D human body scans. In:

INTERNATIONAL CONFERENCE ON 3D BODY SCANNING TECHNOLOGIES, 2, 2011, Lugano. Anais [...]. Lugano: 3D Body.tech, 2011. p. 5101.

ISO. ISO 7250-1: Basic human body measurements for technological design - Part 1: Body measurement definitions and landmarks. International Organization for Standardization, Geneva, Switzerland, 2017.

ISO. ISO 20685-1: 3-D scanning methodologies for internationally compatible anthropometric databases - Part 1: Evaluation protocol for body dimensions extracted from 3-D body scans. International Organization for Standardization, Geneva, Switzerland, 2018.

KEE, D.; KARWOWSKI, W. The boundaries for joint angles of isocomfort for sitting and standing males based on perceived comfort of static joint postures. Ergonomics, v. 44, n. 6, p. 614-648, 2001.

KOUCHI, M. et al. A protocol for evaluating the accuracy of 3D body escâneres. Work, v. 41, n. Supplement 1, p. 4010-4017, 2012.

HIGNETT, S.; MCATAMNEY, L. Rapid Entire Body Assessment (REBA). Applied Ergonomics, v. 31, p. 201-205, 2000.

MCATAMNEY, L.; CORLETT, E. N. RULA: a survey method for the investigation of work-related upper limb disorders. Applied ergonomics, v. 24, n. 2, p. 91-99, 1993.

MICROSOFT. Kinect 360. Disponível em: https://support.xbox.com/pt-BR/browse/xbox-360. Acesso em: 05 set. 2019.

PARK, B.-K.; REED, M. P. Rapid generation of custom avatars using depth cameras. In: INTERNATIONAL CONFERENCE ON 3D
BODY SCANNING TECHNOLOGIES, 3, 2014, Lugano. Anais [...]. Lugano: 3D Body.tech, 2014.

RECFUSION. RecFusion 1.4.6. Disponível em: https://www.recfusion.net/index.php/en/. Acesso em: 05 set. 2019.

SCHWARZ-MUELLER, F.; MARSHALL, R.; SUMMERSKILL, S. Development of a positioning aid to reduce postural variability and errors in 3D whole body scan measurements. Applied ergonomics, v. 68, p. 90-100, 2018.

SHACKEL, B.; CHIDSEY, K. D.; SHIPLEY, Pat The assessment of chair comfort. Ergonomics, v. 12, n. 2, p. 269-306, 1969.

SHU, C. et al. From 3-D Scans to Design Tools. In: INTERNATIONAL CONFERENCE ON 3D BODY SCANNING TECHNOLOGIES, 4, 2013, Lugano. Anais [...]. Lugano: 3D Body.tech, 2013. p.151-156.

SIERRA, I.S.; BARBIERI, B.; SEGALLA, V.; OKIMOTO, M.L.L.R. Requisitos de sistema de escaneamento 3D para pessoas com deficiências motoras. In: Luis Carlos Paschoarelli; Fausto Orsi Medola. (Org.). Tecnologia Assistiva: Pesquisa e Conhecimento II. 1ed. Bauru: Canal 6, 2018, v. 1, p. 11-19.

SIERRA, I.S.; BARBOSA, M.C.; ROCHA, B.; OKIMOTO, M.L.L.R. Avaliação de posturas para antropometria digital considerando tempo, conforto e qualidade da malha obtida. In: CONGRESSO INTERNACIONAL DE ERGONOMIA E USABILIDADE DE INTERFACES HUMANOTECNOLOGIA, 17, 2019, Rio de Janeiro. Anais [...]. São Paulo: Editora Blucher, 2019. v. 6. p. 696-707.

SILVA, F. P.; SALVALAIO, C. L.; KINDLEIN, J. W. Análise Comparativa de Processos de Digitalização 3D para Modelos de Partes Humanas. In: CONGRESSO BRASILEIRO DE PESQUISA E DESENVOLVIMENTO EM DESIGN, 9, 2010, São Paulo. Anais [...]. São Paulo: Anhembi Morumbi, 2010.

SOILEAU, L. et al. Automated anthropometric phenotyping with novel Kinect-based threedimensional imaging method: comparison with a reference laser imaging system. European journal of clinical nutrition, v. 70, n. 4, p. 475, 2016. 
SPAHIU, T.; SHEHI, E.; PIPERI, E. An Attempt for Developing Albanian Anthropometric System within a Pilot Project. In: INTERNATIONAL CONFERENCE ON 3D BODY SCANNING TECHNOLOGIES, 7, 2016, Lugano. Anais [...]. Lugano: 3D Body.tech, 2016. p.269-278.

TARABINI, M. et al. A prototype for the automatic measurement of the hand dimensions using the Microsoft Kinect V2. In: INTERNATIONAL SYMPOSIUM ON MEDICAL MEASUREMENTS AND APPLICATIONS, 2018, Roma. Anais [...]. Roma: MEMEA, 2018. p. 1-6.

VERWULGEN, S. et al. A new data structure and workflow for using 3D anthropometry in the design of wearable products. International Journal of Industrial Ergonomics, v. 64, p. 108-117, 2018.

VITALI, A.; RIZZI, C. Acquisition of customer's tailor measurements for 3D clothing design using virtual reality devices. Virtual and Physical

Prototyping, v. 13, n. 3, p. 131-145, 2018.

\section{Agradecimentos}

Este estudo foi financiado em parte pela Coordenação de Aperfeiçoamento de Pessoal de Nível Superior - Brasil (CAPES) - Código de Financiamento 001. 